\title{
CLASS GROUPS AND AUTOMORPHISM GROUPS OF GROUP RINGS
}

\author{
by KENNETH A. BROWN
}

(Received 13 February, 1985)

\section{Introduction.}

(1.1) This paper is a sequel to [2]. A polycyclic-by-finite group $G$ was there called dihedral free if $G$ contains no subgroup isomorphic to $\left\langle b, a: b^{a}=b^{-1}, a^{2}=1\right\rangle$ whose normalizer has finite index in $G$. It was shown in [2, Theorem $F]$ that, if $R$ is a commutative Noetherian domain, the group ring $R G$ is a prime Noetherian maximal order if and only if $R$ is integrally closed, $G$ is dihedral free, and $G$ has no non-trivial finite normal subgroups. Throughout, $R$ and $G$ will be assumed to satisfy these hypotheses. The main aim of the paper is to study the class group of the maximal order $R G$.

(1.2) Let $S$ be a prime Noetherian maximal order with simple Artinian quotient ring $Q$. By [8, Chapter II, Proposition 2.6], the set of reflexive $S$-ideals in $Q$ forms a group $G(S)$, which is free Abelian with the prime reflexive ideals of $S$ affording a basis. The central class group $\mathrm{Cl}(S)$ of $S$ is the factor of $G(S)$ by the subgroup generated by ideals with central principal generators (see 2.3(2)).

(1.3) Let $R$ be a commutative Noetherian UFD with group of units $R^{*}$, and let $G$ be as above, with FC-subgroup $\Delta$. Write $G_{0}=G / C_{G}(\Delta)$, a finite group, and denote the centre of $R G$ by $C$. Note that $R^{*} \times \Delta$ is a $Z G_{0}$-module under conjugation. Our main findings are as follows.

THEOREM. (i) $\mathrm{Cl}(R G)=H^{1}\left(G_{0}, R^{*} \times \Delta\right)$.

(ii) $\mathrm{Cl}(C)$ embeds in $\mathrm{Cl}(R G)$. The cokernel is the direct sum of finitely many cyclic groups, the class groups $\mathrm{Cl}\left(R G_{P}\right)$ for those reflexive primes $P$ for which the Jacobson radical of $R G_{P}$ is not centrally generated.

(1.4) These facts are proved in Theorem 3.1. Every reflexive ideal of the group rings $R G$ under consideration is principal, generated by an element of $R \Delta$ (3.1(i)). Now $R \Delta$ is a commutative Noetherian domain on which $G_{0}$ acts as ring automorphisms. So the above results arise as special cases of some observations on "class groups under group actions". In case they may have wider application, we have derived the relevant facts in a general context in Section 2, before apply them to group rings in Section 3.

(1.5) The group of $\mathrm{X}$-inner automorphisms $\mathrm{X}-\operatorname{Inn}(S)$ of a prime Noetherian ring $S$ consists of those automorphisms induced by conjugation by a unit $u$ of the simple Artinian quotient ring $Q$ of $S$. Note that $u$ is then normal-that is, $S u=u S$-so, if $S$ is a maximal order, $u S \in G(S)$. If every reflexive $S$-ideal is principal, it follows easily that $\mathrm{X}-\operatorname{Inn}(S) / \operatorname{Inn}(S) \cong \mathrm{Cl}(S)$ (Lemma 4.2). Applying this to group rings in 4.4, we deduce that

$$
\mathrm{X}-\operatorname{Inn}(R G) / \operatorname{Inn}(R G) \cong H^{1}\left(G_{0}, R^{*} \times \Delta\right),
$$

Glasgow Math. J. 28 (1986) 79-86. 
where the notation and hypotheses are those of 1.3. The link between this isomorphism and earlier work of S. Montgomery and D. S. Passman [10, 11] is explored in 4.5.

If $G$ is in addition Abelian-by-finite, the Skolem-Noether theorem implies that the group Aut ${ }_{C}(R G)$ of $C$-algebra automorphisms of $R G$ equals X-Inn $(R G)$, so that

$$
\operatorname{Aut}_{C}(R G) / \operatorname{Inn}(R G) \cong H^{1}\left(G_{0}, R^{*} \times \Delta\right) \text {. }
$$

\section{Class groups under group actions.}

(2.1) For basic facts about maximal orders, see [8]. Throughout Section 2, $S$ will be a prime Noetherian maximal order with Artinian quotient ring $Q$. Let $I$ be a finitely generated right (resp. left) $S$-submodule of $Q$. Call $I$ a right (resp. left) $S$-ideal if $I \cap \mathscr{C}(0) \neq \varnothing$. Let $I^{*}=\operatorname{Hom}_{S}(I, S)=\{q \in Q: q I \subseteq S\}$ (resp. $\left.\{q \in Q: I q \subseteq S)\right\}$. If $I$ is an $S$-S-bimodule this definition is unambiguous, since both sets equal $\{q \in Q: I q I \subseteq I\}[\mathbf{8}$, Chapter I, Proposition 3.1]. The right or left $S$-ideal $I$ is reflexive if $I=I^{* *}$. The operation $I \cdot J=(I J)^{* *}$ makes the set of reflexive $S$-ideals in $Q$ into a group, $G(S)$, which is free Abelian with basis the set $\mathscr{P}$ of prime reflexive ideals of $S$ [8, Chapter II, Prop. 2.6].

Let $\Gamma$ be a group acting on $S$, and put $S^{\Gamma}=\left\{s \in S: s^{\gamma}=s\right.$ for all $\left.\gamma \in \Gamma\right\}$. A subset $X$ of $Q$ is $\Gamma$-invariant if $X^{\gamma}=X$ for all $\gamma \in \Gamma$. If $I$ is a $\Gamma$-invariant $S$-ideal, so is $I^{*}$. Hence $G(S)$ contains the subgroup of $\Gamma$-invariant reflexive $S$-ideals, denoted $G_{\Gamma}(S)$.

Let $l$ be a reflexive $S$-ideal, so that $I=P_{1}^{\varepsilon_{1}} \cdot P_{2}^{\varepsilon_{2}} \cdot \ldots \cdot P_{r}^{\varepsilon_{r}}$, where $P_{i}$ is a reflexive prime and $\varepsilon_{i}= \pm 1$ for all $i$. If $I^{\Gamma}=I$, then $\Gamma$ permutes the $P_{i}$ 's, so that each has a finite $\Gamma$-orbit; and if the $P_{i}$ 's form $t$ such orbits, $I=A_{1} \cdot A_{2} \cdot \ldots \cdot A_{t}$, where, for each $i$, $A_{i}=P_{i_{1}}^{\varepsilon_{i_{1}}} \cdot P_{i_{2}}^{\varepsilon_{i_{2}}} \cdot \ldots \cdot P_{i_{n}(i)}^{\varepsilon_{n(i)}}=\bigcap_{j} P_{i_{j}}^{\varepsilon_{i}}$, the intersection being over the primes in a single orbit. Thus $G_{\Gamma}(S)$ is free Abelian with basis $\left\{\bigcap_{\gamma \in \Gamma} P^{\gamma}: P \in \mathscr{P}, P \Gamma\right.$-orbital $\}$.

(2.2) Let $\operatorname{Prin}_{\Gamma}(S)$ be the subgroup of $G_{\Gamma}(S)$ generated by the principal ideals $S c=c S$ of $S$, with $c \in S^{\Gamma}$. We define the $\Gamma$-normalised class group of $S$ to be the factor $G_{\Gamma}(S) / \operatorname{Prin}_{\Gamma}(S)$, denoted $\mathrm{Cl}_{\Gamma}(S)$.

(2.3) Examples. (1) Let $S$ be commutative and $\Gamma=1$. Then $\mathrm{Cl}_{\Gamma}(S)=\mathrm{Cl}(S)$, the usual divisor class group [1, Ch. VII. \$1, no. 10], [4, Chapter II, §6].

(2) Let $\Gamma=Q^{*}$, the group of units of the quotient ring $Q$ of $S$ (or, more generally, let $\Gamma$ be any subgroup of $Q^{*}$ which generates $Q$ as an algebra over the centre $C$ of $S$ ). Then $S^{\Gamma}=C, \operatorname{Prin}_{\Gamma}(S)$ is generated by the central principal ideals, and $\mathrm{Cl}_{\Gamma}(S)$ is the central class group, studied in [7], for example. In this paper, the central class group will always be denoted by $\mathrm{Cl}(S)$.

(3) If $S^{\Gamma}$ is in the centre $C$ of $S$, and $S$ is a finite $C$-module (or, more generally, $\left.Q=S C^{-1}\right)$, then $\operatorname{Pic}_{S} r(S) \subseteq \mathrm{Cl}_{\Gamma}(S)$, in Frolich's notation: see $[13, \S 37]$.

(4) If $S^{\Gamma} \subseteq C$, and every reflexive $\Gamma$-invariant ideal of $S$ is invertible, then $\mathrm{Cl}_{\Gamma}(S) \subseteq \operatorname{Pic}_{S^{r}}(S)$.

(5) Suppose that $\Gamma=1$. Then $\mathrm{Cl}_{\mathrm{r}}(S)$ is the normalising class group defined by Chamarie: see [7].

(2.4) Let $C_{\Gamma}(S)=\left\{\gamma \in \Gamma: s^{\gamma}=s\right.$ for all $\left.s \in S\right\}$, and set $\Gamma_{0}=\Gamma / C_{\Gamma}(S)$. 
Proposition. Suppose that $S$ is commutative, that every $\Gamma$-invariant reflexive ideal of $S$ is principal, and that $\left|\Gamma: C_{\Gamma}(s)\right|<\infty$ for all $s \in S$. Then $\mathrm{Cl}_{\Gamma}(S) \subseteq H^{1}\left(\Gamma_{0}, S^{*}\right)$. If $\Gamma_{0}$ is finite, this inclusion is an equality.

Proof. Let $K$ be the quotient field of $S^{\Gamma}$. Since each element of $S$ has only finitely many $\Gamma$-conjugates, $K=Q^{\Gamma}$. There is an exact sequence

$$
1 \rightarrow S^{*} \rightarrow Q^{*} \rightarrow \operatorname{Prin}(S) \rightarrow 1 \text {. }
$$

Applying the fixed point functor to this sequence yields

$$
1 \rightarrow\left(S^{*}\right)^{\Gamma} \rightarrow\left(Q^{*}\right)^{\Gamma} \stackrel{\tau}{\rightarrow} \operatorname{Prin}(S)^{\Gamma} \rightarrow H^{1}\left(\Gamma_{0}, S^{*}\right) \rightarrow H^{1}\left(\Gamma_{0}, Q^{*}\right),
$$

[6, \$2.1]. Here, $\left(Q^{*}\right)^{\Gamma}=K^{*}$, and $\left(S^{*}\right)^{\Gamma}=\left(S^{\Gamma}\right)^{*}$. Since the $\Gamma$-invariant reflexive ideals of $S$ are principal, $G_{\Gamma}(S)=\operatorname{Prin}(S)^{\Gamma}$, so that

$$
\mathrm{Cl}_{\Gamma}(S)=\operatorname{Prin}(S)^{\Gamma} / \operatorname{Prin}_{\Gamma}(S) \cong \operatorname{Prin}(S)^{\Gamma} / \operatorname{im} \tau \text {. }
$$

This gives the stated inclusion. If $\Gamma_{0}$ is finite, $H^{1}\left(\Gamma_{0}, Q^{*}\right)=0$ by Hilbert's Theorem 90 [14, Theorem 3.7.2], and so

$$
\mathrm{Cl}_{\Gamma}(S) \cong H^{1}\left(\Gamma_{0}, S^{*}\right)
$$

(2.5) Let $C_{0}$ be an integrally closed Noetherian domain contained in the centre of $S$, with $S$ a finitely generated $C_{0}$-module. An $S$-ideal $I$ is reflexive (as an $S$-ideal) if and only if it is reflexive as a $C_{0}$-lattice in $Q$ [5, Theorem 2.3]. Let $\mathscr{T}$ be the set of height one primes of $C_{0}$. Since $I$ is $C_{0}$-reflexive if and only if $I=\bigcap_{p \in \mathscr{T}} I_{p}[1, \mathrm{Ch}$. VII, $\S 4$, no. 2, Theorem 2], we deduce the well-known first part of Lemma 2.5. The second statement follows from the first by a routine global-local argument.

Lemma. An $S$-ideal $I$ is reflexive if and only if $I=\bigcap_{p \in \mathscr{T}} I_{p}$. Let $K$ be the quotient field of $C_{0}$. If $L$ is a reflexive $C_{0}$-ideal, $(L S)^{* *} \cap K=L$.

(2.6) Proposition. Let $C$ be the centre of $S$, and suppose that $S^{\Gamma} \subseteq C$, with $S$ a finitely generated $S^{\mathrm{r}}$-module.

(a) $S^{\Gamma}$ is an integrally closed Noetherian domain.

(b) The map $i_{0}: G\left(S^{\Gamma}\right) \rightarrow G_{\Gamma}(S): I \rightarrow(I S)^{* *}$ induces a monomorphism $i: \mathrm{Cl}\left(S^{\Gamma}\right) \rightarrow$ $\mathrm{Cl}_{\Gamma}(S)$

Proof. (a) Since $S$ is a maximal order, $C$ is integrally closed. Let $L$ and $K$ be the quotient fields of $C$ and $S^{\Gamma}$ respectively. Since $K \cap C=S^{\Gamma}, S^{\Gamma}$ is integrally closed. Moreover, $S^{\Gamma}$ is Noetherian by [3].

(b) Let $I$ be a reflexive $S^{\Gamma}$-ideal. By Lemma 2.5, $(I S)^{* *} \cap K=I$. So $i_{0}$ is an injection. For reflexive ideals $I$ and $J$ of $S,(I J S)^{* *}=(I S J S)^{* *}=i_{0}(I) \cdot i_{0}(J)$. So $i_{0}$ is a homomorphism. Let $J \in G\left(S^{\Gamma}\right)$ with $i_{0}(J) \in\left\langle\operatorname{Prin}_{\Gamma}(S)\right\rangle$. We claim that $J \in\left\langle\operatorname{Prin}\left(S^{\Gamma}\right)\right\rangle$. Let $(J S)^{* *}=$ $c d^{-1} S$, where $c, d \in S^{\Gamma}$. Therefore $i_{0}(J d)=c S$. Since $(J d S)^{* *} \cap S^{\Gamma}=J d$, we must have $c \in J d$. Hence, $c S=(c S)^{* *} \subseteq(J d S)^{* *}=c S$. Thus, $J d=(J d S)^{* *} \cap S^{\Gamma}=c S^{\Gamma}$. It follows that $J=c d^{-1} S^{\Gamma}$, proving our claim. Therefore $i_{0}$ induces an injection on class groups. 
(2.7) We shall say that a group $\Gamma$ acts locally finitely on a set $A$ if for each $a \in A$ the set $\left\{a^{\gamma}: \gamma \in \Gamma\right\}$ is finite.

THEOREM. Suppose that $S^{\Gamma}$ is in the centre of $S$, and $S$ is a finitely generated $S^{\Gamma}$-module. Let $\mathscr{T}$ be the set of height one primes of $S^{\Gamma}$.

(i) There is an exact sequence

$$
0 \rightarrow \mathrm{Cl}\left(S^{\mathrm{\Gamma}}\right) \stackrel{i}{\rightarrow} \mathrm{Cl}_{\Gamma}(S) \stackrel{\iota}{\rightarrow} \sum_{p \in \mathscr{T}}^{\oplus} \mathrm{Cl}_{\Gamma}\left(S_{p}\right) \rightarrow 0
$$

(ii) Suppose there is a unique $\Gamma$-orbit of height one primes of $S$ lying over each $p \in \mathscr{T}$. Then $\mathrm{Cl}_{\Gamma}\left(S_{p}\right)$ is finite cyclic, of order $e_{p}$, where $e_{p}$ is the index of nilpotency of the radical of $S_{p} / p S_{p}$.

Suppose now that $S$ is commutative, that every $\Gamma$-invariant reflexive ideal is principal, and that $\Gamma$ acts locally finitely on $S$.

(iii) There is a unique $\Gamma$-orbit of height one primes of $S$ lying over each $p \in \mathcal{T}$.

(iv) Let $\Gamma_{0}=\Gamma / C_{\Gamma}(S)$. Suppose that $H^{1}\left(\Gamma_{0}, S^{*}\right)$ is finite. Then for all but finitely many $p \in \mathscr{T}, \mathrm{Cl}_{\Gamma}\left(S_{p}\right)$ is trivial.

Proof. (i)(a) Proposition 2.6 shows that $i$ is a monomorphism.

(b) The map $\tau$ is induced from the map which sends the reflexive $\Gamma$-invariant ideal $l$ of $S$ to $\sum_{p}^{\oplus} I S_{p} \in \sum_{p}^{\oplus} G_{\Gamma}\left(S_{p}\right)$. (Since $I S_{p}=S_{p}$ for all but finitely many $p$, this makes sense.)

(c) $\operatorname{im} i \subseteq \operatorname{ker} \tau$. Let $i$ be a reflexive $S^{\Gamma}$-ideal. Let $p \in \mathscr{T}$. Then $I_{p}$ is $S_{p}^{r}$-reflexive, and so $I_{p}=d S_{p}^{\Gamma}$ for some $d \in I$. Thus $I S_{p}=d S_{p}=I S_{p}^{* *}$. Therefore, if [I] denotes the coset of $I$ in $\mathrm{Cl}\left(S^{\Gamma}\right), \tau i([I])=0$.

(d) $\operatorname{ker} \tau \subseteq \operatorname{im} i$. Suppose that $I$ is a $\Gamma$-invariant reflexive $S$-ideal with $\left[I_{p}\right]=0$ in $\mathrm{Cl}_{\Gamma}\left(S_{p}\right)$ for all $p \in \mathscr{T}$. We have to show that $I=(L S)^{* *}$ for some reflexive $S^{\Gamma}$-ideal $L$. Multiplying $I$ if necessary by a suitable $x \in S^{\Gamma}$, we can assume that $I$ is an ideal of $S$. Thus, for all $p \in \mathscr{T}, I_{p}=S_{p} c_{p}$ for some $c_{p} \in S_{p}^{r}$; and for all but finitely many $p, c_{p}=1$. Define $L=\bigcap_{p} S_{p}^{\Gamma} c_{p} \subseteq \bigcap_{p} S_{p}^{\Gamma=}=S^{\Gamma}$, the last equality by Proposition 2.6. Then $L$ is a reflexive ideal of $S^{\Gamma}$, and for all $p$ in $\mathscr{T}, I_{p}=L S_{p}$, by [1, Ch. VII, $\$ 4$, no. 3]. That is, $I=\bigcap_{p} I_{p}=\bigcap_{p} L S_{p}=$ $(L S)^{* *}$ by Lemma 2.5 .

(e) $\tau$ is onto. In the light of Lemma 2.5 , this follows from [1, Ch. VII, $\S 4$, no. 3].

(ii) Let $p \in \mathscr{T}$. Let $P_{1}, P_{2}, \ldots, P_{n}$ be the $\Gamma$-orbit of prime ideals of $S$ which lie over $p$. By 2.1, $G_{\Gamma}\left(S_{p}\right)=\left\langle\left[\cap P_{i}\right]\right\rangle$ is free Abelian of rank 1 , and there exists $m \geqslant 1$ with $p S_{p}=\left[\left(\cap P_{i}\right)^{m}\right]^{* *}$. Thus $\mathrm{Cl}_{\Gamma}\left(S_{p}\right)$ is cyclic of order $m$, since every ideal of the DVR $S_{p}^{\Gamma}$ is a power of $p S_{p}^{\Gamma}$.

(iii) Let $p \in \mathscr{T}$ and let $\left\{P_{1}, \ldots, P_{m}\right\}$ be a $\Gamma$-orbit of primes of $S$ lying over $p$. Put $I=\bigcap_{i} P_{i}$, so that by hypothesis $I=\beta S$ for some $\beta \in I$. If $\gamma \in \Gamma$ then $I^{\gamma}=I$, and so $\beta^{\gamma}=\beta u$ for some unit $u \in S$. Let $\beta=\beta u_{0}, \beta u_{1}, \ldots, \beta u_{t}$ be the distinct $\Gamma$-conjugates of $\beta$, finite in number by hypothesis. Put $\beta^{\prime}=\prod \beta u_{i}$; thus $\beta^{\prime} \in S^{\Gamma}$ and $\beta^{\prime}$ generates $I^{\prime+1}$. Therefore $I^{t+1} \subseteq p S \subseteq I$. It follows that $\left\{P_{1}, \ldots, P_{m}\right\}$ is the only $\Gamma$-orbit of height one primes containing $p$.

(iv) This follows from (i) and Proposition 2.4 


\section{Group rings.}

(3.1) THEOREM. Let $R$ be a commutative Noetherian UFD, and let $G$ be a dihedral free polycyclic-by-finite group with no non-trivial finite normal subgroups. Let $\Delta$ be the $F C$-subgroup of $G$, let $S=R G$, and denote the centre of $S$ by $C$.

(i) $S$ is a prime Noetherian maximal order in which every reflexive ideal is principal, generated by a $G$-normal element of $R \Delta$.

(ii) Let $G_{0}=G / C_{G}(\Delta)$. Then

$$
\mathrm{Cl}(S)=H^{1}\left(G_{0}, R^{*} \times \Delta\right)=\operatorname{Hom}\left(G_{0} / G_{0}^{\prime}, R^{*}\right) \times H^{1}\left(G_{0}, \Delta\right) .
$$

(iii) Let $\mathscr{T}$ denote the set of height one primes of $C$. Let $\mathscr{P}$ be the set of reflexive prime ideals of $S$. There is a bijection from $\mathscr{P}$ to $\mathscr{T}$ given by $P \rightarrow P \cap C$, for $P \in \mathscr{P}$.

(iv) For each $P \in \mathscr{P}$, define $e_{P}$ by $(P \cap C) S_{p}=\left(P S_{p}\right)^{e_{P}}$, where $p=P \cap C$. Then $e_{P}=1$ for all but finitely many primes $P$, and the sequence

$$
0 \rightarrow \mathrm{Cl}(C) \rightarrow \mathrm{Cl}(S) \rightarrow \sum_{P \in \mathscr{P}}^{\oplus} C_{e_{P}} \rightarrow 0
$$

is exact (where $C_{m}$ denotes the cyclic group of order $m$ ).

Proof. (i) By [2, Theorem F] $S$ is a prime Noetherian maximal order. The reflexive prime ideals of $S$ have the form $S \alpha$, for a $G$-normal element $\alpha$ of $R \Delta$, by [2, Theorem F, Proposition 5.3 and Theorem B]. Thus every reflexive ideal of $S$ has this form, by [8, Ch. II, Proposition 2.6].

(ii) In view of (i), $\mathrm{Cl}(S)$ is the group $\mathrm{Cl}_{G}(R \Delta)$, in the notation of 2.2. Now $\Delta$ is finitely generated, so that $G_{0}$ is finite. Since $\Delta$ is torsion free Abelian [12, Lemma 4.1.6], Proposition 2.4 applies, yielding (ii).

(iii) Let $P=\alpha S$ be a reflexive prime, with $\alpha \in R \Delta$. Then $\alpha R \Delta=P \cap R \Delta$ is a finite intersection of height one primes of $R \Delta$. Since $R \Delta$ is a finitely generated $C$-module, $P \cap C=(P \cap R \Delta) \cap C$ is a height one prime of $C[\mathbf{1}, \mathrm{Ch} . \mathrm{V}, \S 2$, no. 4, Theorem 3]. Since $C$ is integrally closed by Proposition 2.6(a), $P \cap C$ is reflexive. If $p$ is a height one prime of $C$, the prime(s) of $S$ minimal over $p S$ are reflexive by [2, Theorem B], so that the map $\mathscr{P} \rightarrow \mathscr{T}: P \rightarrow P \cap C$ is onto. Injectivity of this map follows from Theorem 2.7 (iii), noting that $R \Delta$ is a finitely generated $C$-module by [12, proof of Lemma 4.1.10].

(iv) This follows from (iii) and Theorem 2.7 .

(3.2) NotE. I don't know whether the exact sequence in 3.1(iv) is always split. It would be very useful, for example with a view to determining $\mathrm{Cl}(C)$, to have a description of the right hand term of the sequence which made the latter easy to calculate.

\section{4. $X$-inner and central automorphisms.}

(4.1) An automorphism $\sigma$ of a prime ring $S$ is $X$-inner if there is a unit $u$ of the Martindale quotient ring $Q_{0}(S)$ with $\sigma(s)=u^{-1} s u$ for all $s \in S$. The set X-Inn $(S)$ of these automorphisms is a normal subgroup of $\operatorname{Aut}(S)$. For details, see $[9, \S 2]$. In this section $S$ 
will always be Noetherian with simple Artinian quotient ring $Q$ and so $Q_{0}(S)=$ $\{q \in Q: I q \subseteq S, 0 \neq I \triangleleft S\}$, and an automorphism of $S$ is X-inner if and only if it is induced by an $S$-normal element of $Q$.

(4.2) LEMmA. Let $S$ be a prime Noetherian maximal order whose reflexive ideals are principal. Then

$$
\mathrm{X}-\operatorname{Inn}(S) / \operatorname{Inn}(S) \cong \mathrm{Cl}(S) .
$$

Proof. Define a map $\theta$ from the group $G(S)$ of reflexive $S$-ideals of the quotient ring $Q$ of $S$ to $\mathrm{X}-\operatorname{Inn}(S) / \operatorname{Inn}(S)$, by $\theta(\alpha S)=\sigma_{\alpha} \operatorname{Inn}(S)$, where $\alpha S=S \alpha$ and $\sigma_{\alpha}$ denotes conjugation by $\alpha$. If $S \alpha=S \beta$, then $\beta=\alpha u$ for some unit $u$ of $S$ and so $\sigma_{\beta}=$ $\sigma_{\alpha} \sigma_{u} \equiv \sigma_{\alpha}(\bmod \operatorname{Inn}(S))$. Thus $\theta$ is a well-defined homomorphism of groups. Clearly, $\operatorname{ker} \theta=\{\alpha S: \alpha$ central $\}$. By definition of $\mathrm{X}-\operatorname{Inn}(S), \theta$ is an epimorphism.

(4.3) Continue the notation of 4.1. Let $C$ denote the centre of the ring $S$. Let $\operatorname{Aut}_{C}(S)=\{\sigma \in \operatorname{Aut}(S): \sigma(c)=c$ for all $c$ in $C\}$, and put $\operatorname{Out}_{C}(S)=\operatorname{Aut}_{C}(S) / \operatorname{Inn}(S)$. Clearly, Aut ${ }_{C}(S) \supseteq \mathrm{X}-\operatorname{Inn}(S)$. Let $N(S)=\left\{q \in Q: q^{-1} S q=S\right\}$. Let $K$ be the quotient field of $C$, and $U(S)$ the group of units of $S$. The following result is a special case of [13, Theorem 37.25]. Only (iii) is not immediately obvious: it follows from the SkolemNoether theorem [13, Corollary 7.2.3].

THEOREM (i) There is a monomorphism of groups

$$
\rho: N(S) / U(S) K^{*} \rightarrow \text { Out }_{C}(S) .
$$

(ii) If $S$ is a prime Noetherian maximal order whose reflexive ideals are principal,

$$
N(S) / U(S) K^{*}=\mathrm{Cl}(S) .
$$

(iii) If $S$ is a finitely generated $C$-module, then $\rho$ is an isomorphism.

(4.4) Theorem. Let $R$ be a commutative Noetherian UFD. Let $G$ be a dihedral free polycyclic-by-finite group with no non-trivial finite normal subgroups. Let $\Delta=\Delta(G)$ and $G_{0}=G / C_{G}(\Delta)$.

(i) $\mathrm{X}-\operatorname{Inn}(R G) / \operatorname{Inn}(R G) \cong H^{1}\left(G_{0}, R^{*} \times \Delta\right)$.

(ii) Let $C$ denote the centre of $R G$. Suppose that $G$ is Abelian-by-finite, so that $\Delta=C_{G}(\Delta)$ is the maximal Abelian normal subgroup of $G$. Then

$$
\operatorname{Out}_{C}(R G) \cong H^{1}\left(G_{0}, R^{*} \times \Delta\right) \text {. }
$$

Proof. (i) By Theorem 3.1(i), the hypothesies of Lemma 4.2 are satisfied with $S=R G$. So the result follows from Lemma 4.2 and Theorem 3.1(ii).

(ii) By [12, proof of Lemma 4.1.10], $R \Delta$ is a finitely generated $C$-module, so that $R G$ is a finitely generated $C$-module. Theorems 3.1 (ii) and 4.3 yield the desired conclusion.

(4.5) In [10] and [11], S. Montgomery and D. S. Passman obtained a description of the group $W$ of all those $\mathrm{X}$-inner automorphisms of the prime group algebra $K G$ which normalise the trivial units of $K G$ (for an arbitrary group $G$ and field $K$ ). Here we relate 
their result to 4.4. In doing so, we make explicit the isomorphisms of 4.4(i) and (ii). We follow the notation of $[\mathbf{1 0}, \mathbf{1 1}]$, letting $l$ denote the group of inner automorphisms of $G$ viewed as automorphisms of $K G$, so that $I=W \cap \operatorname{Inn}(K G)$ by [10, Proposition 1]. In this paragraph only, $C$ denotes $\{\sigma \in \operatorname{Aut}(G): \sigma$ centralises a subgroup of finite index in $G\}$, and $S$ the automorphisms of $K G$ of scalar type. That is, $\sigma \in S$ if and only if there is a linear character $\lambda: G \rightarrow K$ with $C_{G}(x) \subseteq \operatorname{ker} \lambda$ for some $x \in \Delta=\Delta(G)$, and $\sigma\left(\sum r_{g} g\right)=$ $\sum r_{g} \lambda(g) g$.

Then $C, I$ and $S$ are normal subgroups of $W, W=C I \cap S$, with $C \cap I$ equal to the group of inner automorphisms induced by $\Delta$, and $W / I \cong(C / C \cap I) \times S$ is periodic Abelian [11, Theorem 3].

Suppose that $\Delta$ is finitely generated, so that $B=C_{G}(\Delta)$ has finite index in $G$. The proof of [11, Lemma 2] shows that $B=C_{G}(y)$ for some $y \in \Delta$. It follows that $S \cong \operatorname{Hom}\left(G / B, K^{*}\right)=H^{1}\left(G / B, K^{*}\right)$.

We turn now to $C$, again assuming that $\Delta$ is finitely generated and continuing to write $B=C_{G}(\Delta)$. It is not hard to show that $C=\{\sigma \in \operatorname{Aut}(G): \sigma$ centralises $B$ and $G / \Delta\}$, using arguments similar to those in [11, proof of Lemma 1]. Let $C_{0}=\{\sigma \in \operatorname{Aut}(G): \sigma$ centralises $\Delta$ and $G / \Delta\}$. Then $C \subseteq C_{0}$, and $C_{0}$ is torsion free Abelian, as in [11, proof of Lemma 1(iii)]. Moreover, $C / C \cap I$ is the torsion subgroup of $C_{0} / C \cap I$-this is an easy consequence of [11, Lemma $1\left(\right.$ ii)] . But $C_{0} / C \cap I=H^{1}(G / \Delta, \Delta)$, by $[\mathbf{6}, \S 3.5$, Proposition 5].

We have an exact sequence of Abelian groups

$$
0 \rightarrow H^{1}(G / B, \Delta) \stackrel{\inf }{\rightarrow} H^{1}(G / \Delta, \Delta) \stackrel{\text { res }}{\rightarrow} H^{1}(B / \Delta, \Delta),
$$

where inf and res denote the inflation and restriction maps [6, page 93]. Since $H^{1}(B / \Delta, \Delta)=\operatorname{Hom}(B / \Delta, \Delta)$ is torsion free [6, page 45], $H^{1}(G / B, \Delta)$ is the torsion subgroup of $H^{1}(G / \Delta, \Delta)$. Therefore,

$$
C / C \cap I \cong H^{1}(G / B, \Delta) .
$$

Summarising then, we state the following, for comparison with 4.4.

Theorem (Montgomery and Passman). Let $G$ be a group with $\Delta(G)$ finitely generated and torsion free. Put $G_{0}=G / C_{G}(\Delta)$. Let $K$ be a field, and let $W$ and $I$ be as above. Then

$$
W \operatorname{Inn}(K G) / \operatorname{Inn}(K G) \cong W / I \cong H^{1}\left(G_{0}, K^{*} \times \Delta\right) .
$$

Suppose that $G$ is polycyclic-by-finite. By Theorem 3.1(i), every normal element of $K G$ has the form $\alpha \beta$, where $\beta$ is a unit of $K G$ and $\alpha$ is a $G$-normal element of $K \Delta(G)$. It follows easily that

$$
\mathrm{X}-\operatorname{Inn}(K G)=W \operatorname{Inn}(K G) .
$$

So in this case the above theorem follows from 4.4 .

ACKNowledgement. I would like to thank Jim Howie for several helpful discussions. 


\section{REFERENCES}

1. N. Bourbaki, Algèbre Commutative (Hermann, 1965).

2. K. A. Brown, Height one primes of polycyclic group rings, J. London Math. Soc., to appear.

3. D. Eisenbud, Subrings of Artinian and Noetherian rings, Math. Ann. 185 (1970), 247-249.

4. R. M. Fossum, The Divisor Class Group of a Krull Domain (Springer-Verlag, 1970).

5. O. Goldman, Quasi-equality in maximal orders, J. Math. Soc. Japan 13 (1961), 371-375.

6. K. W. Gruenberg, Cohomological Topics in Group Theory (Springer-Verlag, 1970). 1982).

7. L. Le Bruyn, Class groups of maximal orders over Krull domains, preprint (Antwerp,

8. G. Maury and J. Raynaud, Ordres Maximaux au Sens de K. Asano (Springer-Verlag, 1980).

9. S. Montgomery and D. S. Passman, Crossed products over prime rings, Israel J. Math. 31 (1978), 224-256.

10. S. Montgomery and D. S. Passman, X-inner automorphisms of group rings, Houston J. Math. 7 (1981), 395-402.

11. S. Montgomery and D. S. Passman, X-inner automorphisms of group rings II, Houston J. Math. 8 (1982), 537-544.

12. D. S. Passman, The Algebraic Structure of Group Rings (Wiley-Interscience, 1977).

13. I. Reiner, Maximal Orders (Academic Press, 1975).

14. D. J. Winter, The Structure of Fields (Springer-Verlag, 1974).

Department of Mathematics

UNIVERSITY OF GLASGOW

UNIVERSITY GARDENS

GLASGOW

G12 8QW 